\title{
DOUBLE-SIDED MICROPILLAR ARRAYS FOR CELL CONTRACTION FORCE MAPPING USING OPTICAL MOIRÉ METHOD
}

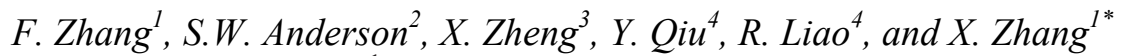 \\ ${ }^{1}$ Boston University, USA \\ ${ }^{2}$ Boston University Medical Center, USA \\ ${ }^{3}$ Lawrence Livermore National Laboratory, USA \\ ${ }^{4}$ Brigham and Women's Hospital, USA
}

\begin{abstract}
The mapping of cellular traction forces is fundamental to deeply understanding a wide range of cellular functions and behaviors. The moiré phenomenon has been leveraged as a visualization tool for cellular force mapping, yielding both a magnification effect as well as the capacity for whole-field force measurements. This paper reports the development of a doublesided micropillar array (DMPA) made of PDMS for use in moirébased cellular force analyses. Herein, the fabricated DMPAs were validated by mapping neonatal rat ventricular myocyte (NRVM) contraction forces. The advantages of the DMPA-based approach yield an improved moiré contrast and enable efficient moiré pattern generation.
\end{abstract}

\section{INTRODUCTION}

Cellular traction forces and the mechanical interaction of cells with their local microenvironment and extracellular matrix (ECM) have been found to be essential to a variety of cell functions ranging from migration to proliferation and apoptosis, among others [1]. In addition, cellular traction forces are fundamental to a variety of processes in both the maintenance of health as well as the development of disease such as, for example, wound healing and inflammation, in addition to cancer invasion and metastasis [2]. In order to study these processes, microfabricated polymeric pillar arrays have been developed and successfully employed for precisely mapping cellular traction forces [3]. Inherent to the application of fabricated micropillar arrays as tools for studying cellular forces is the need for tracking and monitoring the deflections of each individual micropillar as these pillar deflections are employed to calculate the displacement vectors of the cellular traction forces. Since the deflection of pillars is traditionally measured with conventional optical microscopy using a fixed numerical aperture of the objective lens, small-scale deflections or motions of individual sensing units that are in the sub-micrometer to nanometer range may readily fall below the limits of detection, limiting the range of measurable cell force. In addition, conventional optical microscopy is time-consuming and requires complex algorithms in order to track and interpret individual deflections and motions of the high-density micropillar arrays and therefore cannot meet the demands for automated, real-time monitoring of cellular and sub-cellular behaviors. This lack of efficiency precludes the capacity for high-throughput whole-field cellular force mapping of multiple cells in a large field-of-view.

The moiré phenomenon occurs when two periodic patterns overlap to produce a virtual periodic pattern. This phenomenon has previously been employed for measuring strain and deformation, using the moiré effect produced by the specimen and reference gratings to magnify the surface deformations and create a contour map which is related to surface displacement [4]. We have previously leveraged the moiré phenomenon in order to develop an optomechanical approach to cellular force mapping which yields several distinct advantages when compared to conventional approaches, including its marked sensitivity to micropillar displacements, enabling a dramatic expansion of the range of measurable forces as well as high-throughput, real-time wholefield force mapping [5]. In the optical moiré setup, cells are seeded onto the top surface of the micropillar array in a culture dish, while a reference substrate featuring an identical pattern is located below the culture dish on a translation axis stage. The moiré pattern generated by these two substrates is obtained and the displacements of the pillars are recorded in the moire pattern and may be readily retrieved using a variety of image analysis algorithms, thereby yielding cellular force maps. The distance between these two parallel patterns plays a critical role in the image quality of the moiré pattern and demands precise alignment in order to generate a single moiré pattern with appropriate contrast. Furthermore, since the reference substrate is exposed to air and PDMS is inherently sticky, dust and particulate residue is often found on the reference substrate and any such discrepancy between two substrates results in a degradation of the image

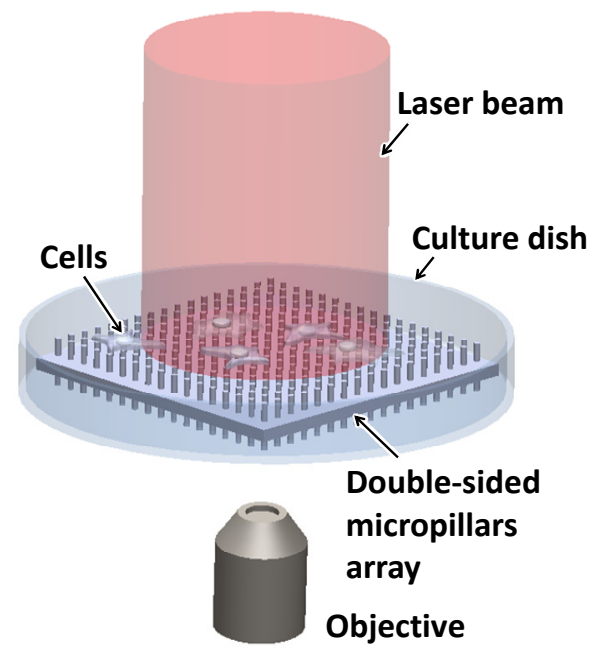

Figure 1: Optical configuration for two-dimensional moirébased cell force mapping using double-sided micropillar arrays. (Size of the cells and pillars are exaggerated as compared to the light beam.)

quality, ultimately affecting the accuracy of cellular force mapping. In order to address the aforementioned existing limitations of the two-dimensional moiré-based approach, the method reported in this work utilizes a double-sided micropillar array (DMPA) in order to map the cellular force distribution, as shown in Figure 1.

\section{RESULTS}

The DMPA was fabricated using a two-sided replica molding process involving two molds, as illustrated in Figure 2. In this 
work, the micropillar arrays on the cell culture side were fabricated with a micropillar diameter of $2 \mu \mathrm{m}$, micropillar height of $6 \mu \mathrm{m}$, and a center-to-center distance of $4 \mu \mathrm{m}$; the micropillar arrays on the reference side of the DMPA featured an identical diameter and center-to-center distance as the cell culture side but a height of 2 $\mu \mathrm{m}$. Conventional photolithography techniques were used to

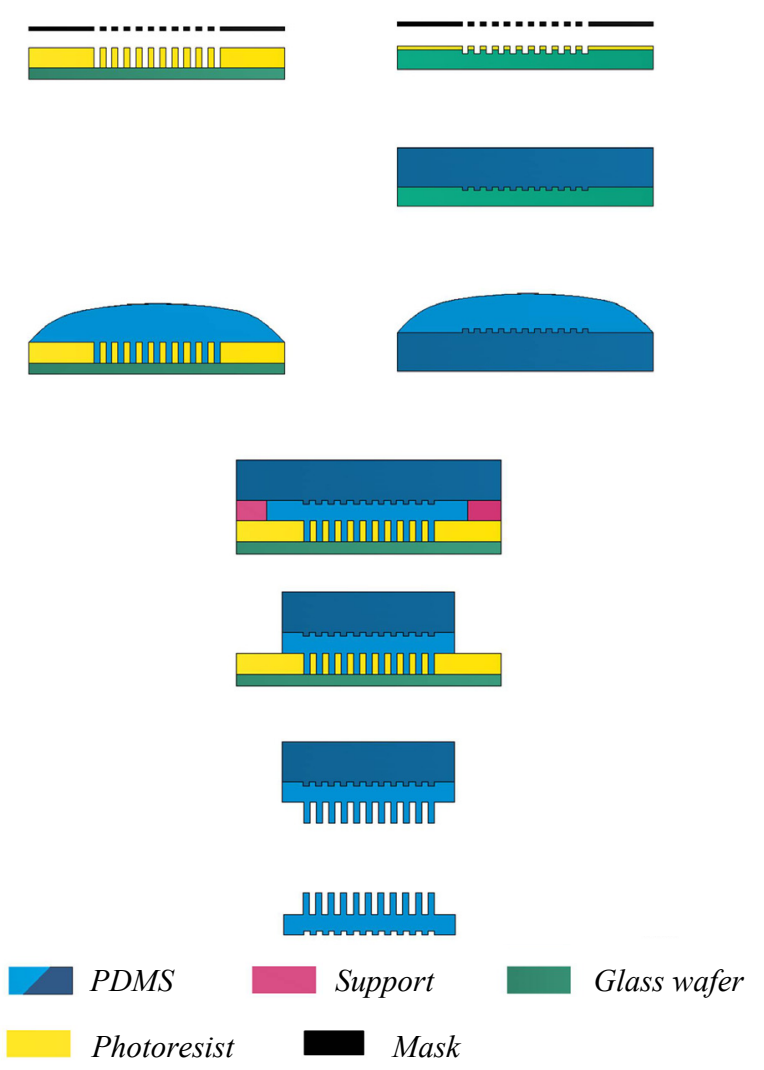

Figure 2: Process flow for PDMS pillar fabrication. The fabrication employs conventional photolithography and a twostep PDMS molding process with a final double casting step which yields double-sided pillar arrays for force mapping.

fabricate the AZ9260 photoresist molds. After replica molding using a glass wafer covered by the patterned photoresist, a PDMS mold with a lower aspect ratio was formed as the mold for the reference pattern side of the DMPA. Both the PDMS mold and the photoresist mold were silanized using (tridecafluoro-1,1,2,2tetrahydrooctyl)-1-trichlorosilane for $10 \mathrm{~h}$ under vacuum in a desiccator to assist with the subsequent release of the two molds and the DMPA. During this process, four pieces of cover glass were used as the supporting structures. After applying $50 \mu \mathrm{L}$ of uncured PDMS prepolymer on one side of each cover glass, the glass wafers were turned over and attached to the four edges along the periphery of the photoresist mold. The photoresist mold with the cover glass was cured at $65^{\circ} \mathrm{C}$ for $30 \mathrm{~min}$ to fix the cover glass, ensuring that is served as a support structure and spacer during the following molding process. PDMS prepolymer was then poured over the surface of both the photoresist and PDMS molds; after 30 min of degassing, the two molds were carefully brought together and gradually attached from one side to another, in order to avoid any bubble formation. The relative pattern orientation of the two molds was precisely controlled using the alignment of the patterned photoresist and the PDMS mold, while the thickness of the supporting glass determined the distance between two periodic patterns. After curing for $2 \mathrm{~h}$ at $65{ }^{\circ} \mathrm{C}$ (corresponding to Young's modulus of PDMS of $1 \mathrm{MPa}$ ), the DMPA was released from the molds and attached to a glass slide for subsequent surface treatment and cell seeding., as shown in Figure 3.
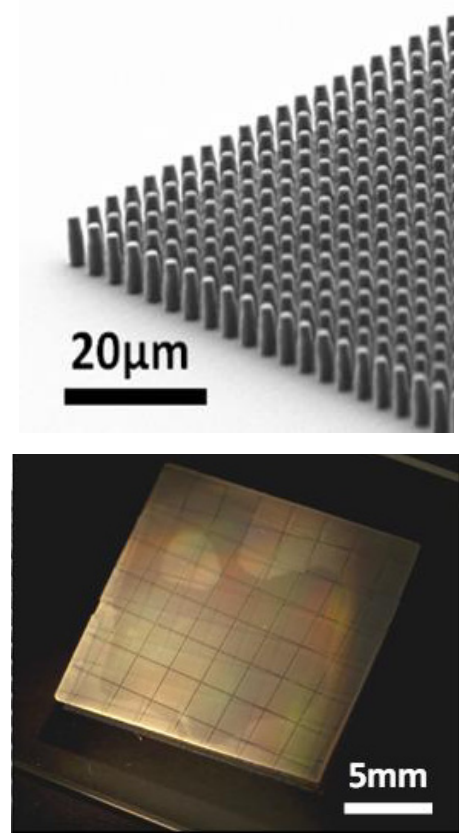

Figure 3: SEM image of pillars at the periphery of an array (top). Double-sided PDMS micropillar array attached to a glass slide (bottom).

Herein, as a validation of the optomechanical DMPA-based cell force mapping approach, we employed neonatal rat ventricular myocytes (NRVM). With a cell size of $10-30 \mu \mathrm{m}$ in diameter, the NRVMs are capable of spontaneously generating forces in the range of $10-200 \mathrm{nN}$; compared to adult rat cardiac myocytes, NRVMs are small in size and generate relatively weak contraction forces. With the advantages of an inherent magnification factor and real-time, whole-field monitoring capacity, the moiré cell force mapping method is an ideal tool for the high-throughput study of NRVMs.

Following fabrication, the DMPAs were prepared for cell attachment using a microcontact printing technique. DMPAs were initially treated by $\mathrm{O}_{2}$ plasma in a Plasma Asher at $300 \mathrm{sccm}$ flow rate and $150 \mathrm{~W}$ for $2 \mathrm{~min}$. PDMS stamps were immersed in a laminin solution $(50 \mu \mathrm{g} / \mathrm{mL})$ for $1 \mathrm{~h}$ and were subsequently placed in conformal contact with the plasma treated DMPAs, thereby coating the top surfaces of the pillars with laminin in order to ensure that subsequently seeded cells adhered only to the top surface of the micropillar arrays. One hundred thousand NRVMs per $\mathrm{mL}$ were seeded onto the cell culture side of the DMPAs and cultured for 2-3 days, allowing the cells to adhere and spread out onto the micropillars. After 2-3 days of culturing, the samples were washed using fresh culture medium to remove the dead cells and the cellular forces analyzed.

\section{RESULTS}

The algorithm for mapping cellular force from the deformed 
moiré patterns involves decoupling the phase distribution from the distorted moire fringe patterns. The displacement information of the pillars is encoded within the aforementioned, cell-induced distortion of the moiré pattern, requiring a series of imageprocessing steps to derive cell force maps. For a moiré pattern, the light intensity may be expressed as:

$$
I(x, y)=a(x, y)+b(x, y)\left[\exp \left(2 \pi j f_{0} x+\Phi(x, y)\right)\right]
$$

where $a(x, y)$ is the background intensity, $b(x, y)$ is the amplitude of the moiré pattern, $f_{0}$ is the frequency of the moiré pattern and the phase information, and $\Phi(x, y)$ contains the deformation of the moiré pattern. In order to compute the contraction force distribution from the moiré patterns, a Fourier transform was applied to the moiré patterns. After frequency filtering, the frequency carrying the information of the moiré fringes was selected from the original frequency map. Subsequently, a reverse Fourier transform was carried out on the filtered frequency domain image and a matrix of complex numbers was obtained. The phase of each complex element in the matrix is calculated and, subsequently, a phase unwrapping algorithm was applied to the wrapped phase images to eliminate $2 \pi$ jumps, yielding unwrapped phase images. By comparing the unwrapped phase map with the phase map derived before cell spreading and contracting, the average ascending trend could be subtracted and the deformation field derived. The deformation field is linearly correlated with the force distribution described as:

$$
u(x, y)=d \Phi(x, y) / 2 \pi
$$

which allows for the simple derivation of a cellular force map using the following relationship:

$$
F(x, y)=k \cdot u(x, y)
$$

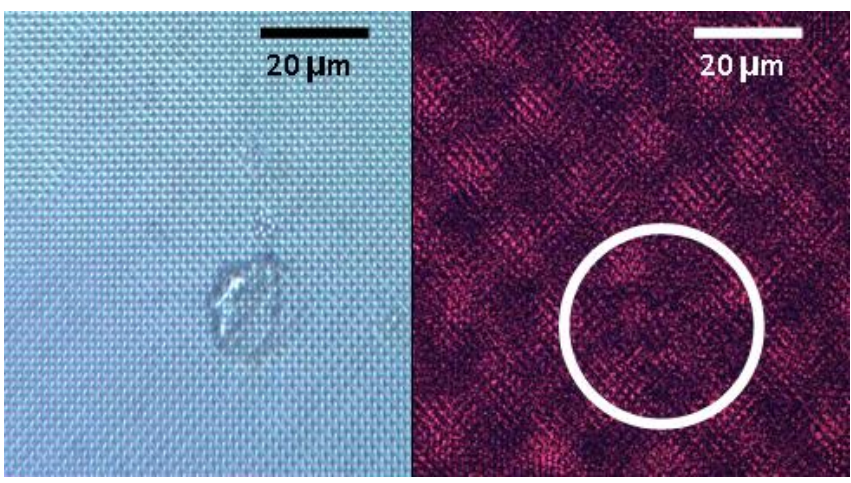

Figure 4: Microscopic image of neonatal rat ventricular myocyte cell cultured on the double-sided pillar arrays (left). Moiré pattern generated by the deformed cell (right). Circle indicates the distorted moiré grid. Note that the pillar deformation is readily visible in the moiré pattern due to the inherent magnification effect of the moiré phenomenon.

Following seeding of the DMPAs with the neonatal rat ventricular myocytes, pillars on the cell culture side of the substrate were deformed by exertion of cellular traction forces (Figure 4). Given the deformation of one of the matched patterns of the DMPAs, distorted moiré patterns were formed, yielding the capacity for cellular force mapping as detailed above (Figure 4).
Due to the magnification of moiré effect, though the deformation of the pillars in the microscopic image is unapparent to visual inspection, the magnified deformation of one grid within the moire pattern (represented by dashed circle) is readily apparent using the $40 \mathrm{X}$ objective.

Utilizing the image processing algorithm detailed above, the deformed moire pattern demonstrated in Figure 4 was converted into a cell force map shown in Figure 5. In the case of the moiré force mapping method, the peak value of the NRVM contraction force was found to approximate $120 \mathrm{nN}$, with the maximal force located at the left side of the cell body. Figure 5 also illustrates the force map generated by calculating each pillar's displacement using the conventional microscopy-based approach which serves as a reference standard. The peak value of the cellular force using this approach was also found to be at the leftward periphery of the cell with a value of approximately $132 \mathrm{nN}$. Compared to this direct measurement approach using the conventional microscopy image, the cellular force derivation using the moiré mapping method is consistent with this reference standard method in force magnitude, but is significantly less time-consuming as the force map is generated from a larger field of view without observing individual pillars, a direct result of the magnification effect. This capacity to efficiently derive whole-field cellular forces in real-time offers a significant advantage compared to the conventional approach.
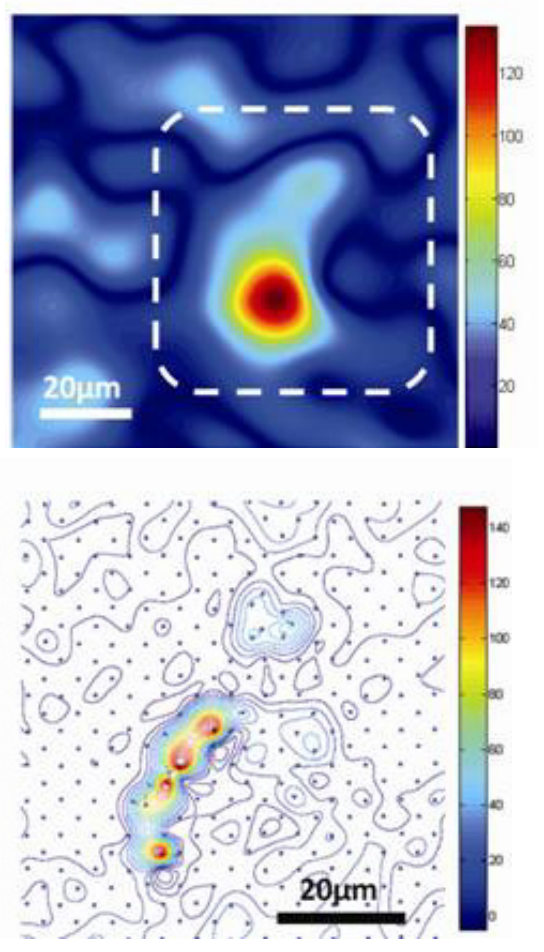

Figure 5: Comparison of force map generated by moiré method (top) and conventional microscopic image analysis (bottom).

\section{CONCLUSION}

In summary, this work reports a double sided micropillar array capable of generating moiré patterns that map cellular force distribution without utilizing any additional reference substrate or light source. These unique arrays improve the efficiency of moiré pattern generation as well as optimize the moiré contrast. NRVM 
were employed to validate the DMPA-enabled optomechanical approach, demonstrating the capacity for efficiently generating accurate cellular force maps. The double-sided structure reported in this work has the potential for enabling the facile integration of moiré-based cell force mapping with microfabricated cell culture environments or lab-on-a-chip devices.

\section{ACKNOWLEDGEMENT}

The authors would like to express thanks and gratitude for the contributions from the staff of the Photonics Center of Boston University. We also thank Xiaoguang Zhao, Erin Roberts, Paul Lou, and other members at the Laboratory for Microsystems Technology (LMST), Boston University, for thoughtful discussions and help with the experiments.

\section{REFERENCES}

[1] V. Vogel and M. Sheetz, "Local Force and Geometry Sensing Regulate Cell Functions", Nature Reviews. Molecular Cell Biology, 7, 265 (2006).
[2] Z. Li, J. Song, G. Mantini, M. Lu, H. Fang, C. Falconi, L. Chen, and Z. Wang, "Quantifying the Traction Force of a Single Cell by Aligned Silicon Nanowire Array", Nano Letters, 9, 3575 (2009).

[3] M. T. Yang, J. Fu, Y. K. Wang, R. A. Desai, and C. S. Chen, "Assaying Stem Cell Mechanobiology on Microfabricated Elastomeric Substrates with Geometrically Modulated Rigidity", Nature Protocols, 6, 187 (2011).

[4] B. Han, D. Post, and P. Ifju, "Moiré Interferometry for Engineering Mechanics: Current Practices and Future Developments", The Journal of Strain Analysis for Engineering Design, 36, 101 (2001).

[5] X. Zheng and X. Zhang, "An Optical Moiré Technique for Cell Traction Force Mapping", Journal of Micromechanics and Microengineering, 18, 125006 (2008).

\section{CONTACT}

*X. Zhang, tel: + 1.617.358.2702; xinz@bu.edu 